\title{
Application of GPS to Enable Launch Vehicle Upper Stage Heliocentric Disposal
}

\author{
Evan J Anzalone, Ph.D., NASA/Marshall Space Flight Center \\ T. Emerson Oliver, NASA/MSFC/Dynamic Concepts Incorporated
}

\section{BIOGRAPHIES}

Evan Anzalone, Ph.D. is the Guidance and Navigation Team Lead for the Guidance, Navigation, and Mission Branch within the Spacecraft and Vehicle Systems Department at NASA/Marshall Space Flight Center. He earned his PhD in 2013 from the Georgia Institute of Technology with his thesis which focused on the application of Model-based Systems Engineering to the development of Navigation Technologies, through design of the Multi-spacecraft Autonomous Positioning System. He has been at MSFC since 2010, and is currently supporting B1B Navigation Development as well as the Software-driven Navigation for Station experiment to be hosted on the SCaN Testbed on ISS in early 2018.

T. Emerson Oliver, M.S, is the SLS Navigation Team Lead. He has been at MSFC for 11 years, and received his Master of Science degree from Mississippi State University in Aerospace Engineering with his thesis, "Navigation System Design with Application to the Ares I Crew Launch Vehicle and Space Launch Systems. " He has extensive experience in the application of inertial navigation algorithm development, requirements analysis, and hardware insight for launch vehicle applications. These applications range from on-ground attitude determination, inertial navigation, and state estimation. In addition to algorithm development, he has a large amount of hardware experience and is leading an effect processing results from Hardware-in-the-Loop testing of the SLS Inertial Navigation Unit on a 6DOF table in support of model validation.

\begin{abstract}
To properly dispose of the upper stage of the Space Launch System, the vehicle must perform a burn in Earth orbit to perform a close flyby of the Lunar surface to gain adequate energy to enter into heliocentric space. This architecture was selected to meet NASA requirements to limit orbital debris in the Earth-Moon system. The choice of a flyby for heliocentric disposal was driven by mission and vehicle constraints. This paper describes the SLS mission for Exploration Mission -1, a high level overview of the Block 1 vehicle, and the various disposal options considered. The research focuses on this analysis in terms of the mission design and navigation problem, focusing on the vehicle-level requirements that enable a successful mission. An inertial-only system is shown to be insufficient for heliocentric flyby due to large inertial integration errors from launch through disposal manuever while on a trans-lunar trajectory. The various options for aiding the navigation system are presented and details are provided on the use of GPS to bound the state errors in orbit to improve the capability for stage disposal. The state estimation algorithm used is described as well as its capability in determination of the vehicle state at the start of the planned maneuver. This data, both dispersions on state and on errors, is then used to develop orbital targets to use for meeting the required Lunar flyby for entering onto a heliocentric trajectory. The effect of guidance and navigation errors on this capability is described as well as the identified constraints for achieving the disposal requirements. Additionally, discussion is provided on continued analysis and identification of system considerations that can drive the ability to integrate onto a vehicle intended for deep space.
\end{abstract}

\section{INTRODUCTION}

The upcoming NASA Space Launch System (SLS) Exploration Mission-1 (EM-1) (Gerstenmaier) is the first flight of the Block 1 configuration of the launch vehicle, with the primary purpose of demonstrating flight and ground systems for future flights, as well to place the Orion Multi-Purpose Crew Vehicle into a Distant Retrograde Orbit about the Moon. This will allow for deep space certification and demonstration of the crew module prior to future crewed flights. A high-level architecture diagram of the current iteration of the planned mission is provided in Figure 1. This lays out the primary maneuvers and sections of the flight from orbital ascent, Low Earth Orbit insertion, Trans-Lunar Injection, Trajectory Correction Maneuvers, Lunar Fly-By, and Powered Return back to Earth, and final splashdown.

This research was primarily conducted in support of Vehicle Management within the SLS Program, particularly focused on the Navigation aspects of the mission, with a focus on development and assessment of vehicle-level requirements and integrated 
Core vehicle performance. The SLS Core Stage consists of 4 RS-25 Space Shuttle Main Engines (SSMEs) with 2 5-segement Solid Rocket Boosters (SRBs) that provide additional thrust during initial ascent. The primary requirement of the core stage is to insert the payload consisting of the upper stage and Orion into the defined orbit to enable the TLI burn and eventual lunar fly-by. The launch stage's mission is considered complete and successful with the insertion of Orion onto a trans-lunar orbit. The upper stage, interim Cryogenic Propulsion Stage (iCPS), is being developed by United Launch Alliance (ULA), based on Delta Common Second Stage (DCSS) heritage, utilizing one RL-10B engine. This stage is essentially being considered COTS hardware, with the NASA team providing insight and requirements design and assessment to asses integrated vehicle performance, and assessing design sensitivities to the Core vehicle's capability. This research will focus on development of navigation requirements in order to meet the successful completion of the mission given Core Stage insertion capability, with a focus on the last step of the ICPS mission: stage disposal.

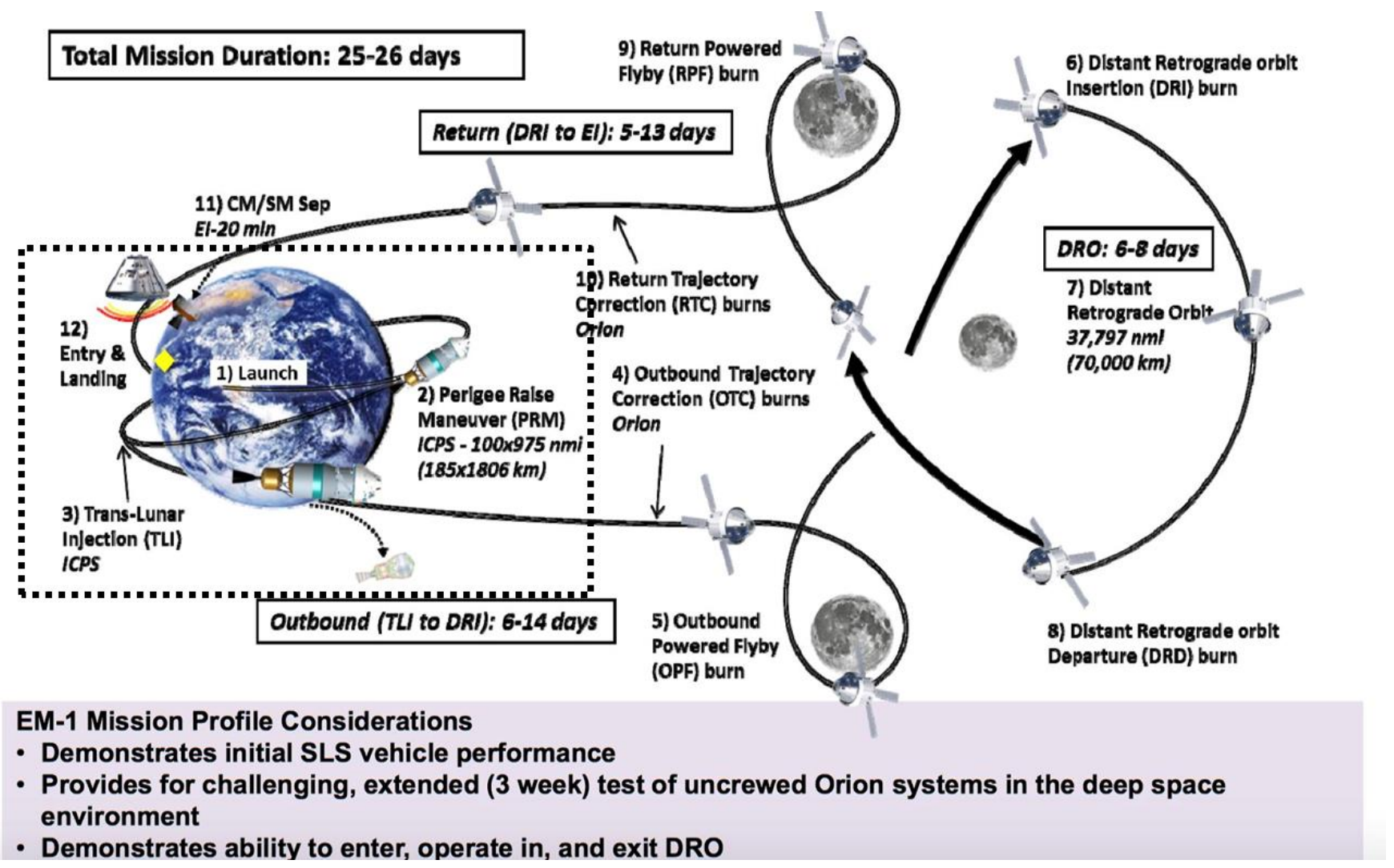

\section{NEED FOR DISPOSAL}

Figure 1: SLS EM-1 Reference Mission (Gerstenmaier)

In addition to placing the payload on a trans-lunar trajectory, the iCPS must properly be disposed of to meet NASA STD 8791-14((NASA). This inter-agency agreement defines constraints for what to do with spent stages and satellites at their end of life. NASA-STD-8719-14 was developed to address and limit the amount of ortibal debris in the Earth-Moon neighborhood to reduce the risks of impacts to future missions and help to reduce the amount of orbital debris. Additionally, this document has been levied to require satellite missions in LEO to have end-of-life plans and de-orbit capabilities for higher altitudes where the orbits are naturally stable with lengthy natural decay periods (100s of years). Several options are laid out, including: controlled breakup, plaentary impact (Earth or Lunar surface), and insertion into heliocentric space. Each of these options carries specific criteria for measuring success, constraints for implementation, and unique challenges.

For the SLS missions, the core stage is disposed of through re-entry and eventual splashdown in the Atlantic Ocean. A large amount of Monte Carlo analsyis is performed prior to flight to ensure no impact to populated areas with a high degree of confidence. While traditionally Lunar-bound missions have allowed for Lunar impact, this still requires high confidence of avoiding histroic sites and active research areas (for example mirrors placed on the Lunar surface by Apollo astroanuts). For these purposes, a Lunar impact was shown to be highly undesirable. A direct burn into heliocentric orbit was not considered due to the existing design of the EM-1 trajectory. Instead, requirements were levied on the uppper stage for it to perform a heliocentric burn to enable Lunar Fly-by. The ability to meet this mission is heavily dependent on the GNC system of the 
vehicle and on the Earth-Moon geometry. This is primarily due to the limited operational lifteime of the upper stage and lacking capabiilty for orbital trajectory maneuvers. As such, the stage must perform a burn placing it on a flyby trajectory for several days of coast after the maneuver. At the Lunar interface, the vehicle must then fly through a pre-defined altitude window in order to gain enough velocity to enter into a heliocentric trajectory.

\section{INERTIAL-ONLY CAPABILITY}

The capability to successfully dispose is a product of the integrated vehicle design and performance. The SLS takes advantage of flight heritage inertial navigation systems to support vehicle Guidance, Navigation, and Control (GNC) functions. As such the capability to successfully design and execute a manuever in High Earth Orbit is defined by both the state dispersions (position, velocity, time) as well as dispersions in navigation errors (errors between truth and navigated states). Due to this tight coupling, these errors can be traced back through Trans-Lunar Injection capability and further to Ascent Insertion performance of the Core Stage. In order to assess this scenario, a highly detailed 6DOF model was developed in order to fully capture the vehicle's capability, which included notional guidance and navigation algorithms. Additionally, all analysis in this work was performed using a notional mission and navigation error budget based on publicly available sources (Honeywell Aerospace Electronic Systems) of a state-of-the-art inertial navigation system. The trajectory used for analysis is also notional, and does not represent the final mission.

To assess the disposal capability, the MAVERIC (Hanson) vehicle simulation was used to capture the insertion capability of the core vehicle for a given launch date. This included notional models of the upper stage navigation system to capture its navigation error at the insertion into Low Earth Orbit. It's initial truth state dispersions are a function of the Core vehicle's GNC capability, capturing the coupling between the navigated state fed into the guidance targeting algorithms and the unknown navigation error (to the onboard algorithms). For ascent capability, the insertion capability is more driven by navigation errors rather than the ability to meet an orbital target. Similar guidance algorithms to those used during ascent were implemented and used for the maneuvering up to and including the Trans-Lunar Injection manuever. This mission included a one orbit coast for vehicle checkout and additional coasting past TLI to allow for MPCV separation, allowing for additional separation between the vehicle elements to reduce risk of re-contact during further operations. As such, the final disposal burn is performed at an altitude of $25,000 \mathrm{~km}$ several hours into the mission.

This high altitude is due to the vehicle being on a trans-lunar trajectory and rapidly exiting Earth's sphere of influence. Due to the usage of un-aided inertial systems and long vehicle timeline between ground alignment and final maneuver, the errors grow considerably. In addition to intrinsic sensor errors terms, the nonlinearities of the state integration, principally in terms of orbital dynamics and gravity modeling (and the instabilities associated with predicting gravity with an errored state) begin to play a much larger part in integrated errors. An overview of the navigation error growth over the orbital phase of this missions is shown in Figure 2. These results present a Monte Carlo-based analysis of the navigation performance for dispersed vehicles and sensor error states. The errors are represented in an Radial-Tangential-Normal frame defined by the vehicle's state. In these timelines, the TLI maneuver occurs around 5000 seconds. Interestingly, these plots clearly show the effects of orbital dynamics on the inertial error growth. Primarily, this is an effect of the vehicle flying on a non-elliptical orbit, and the dispersions shift between position and velocity states due to being at perigee or apogee. 

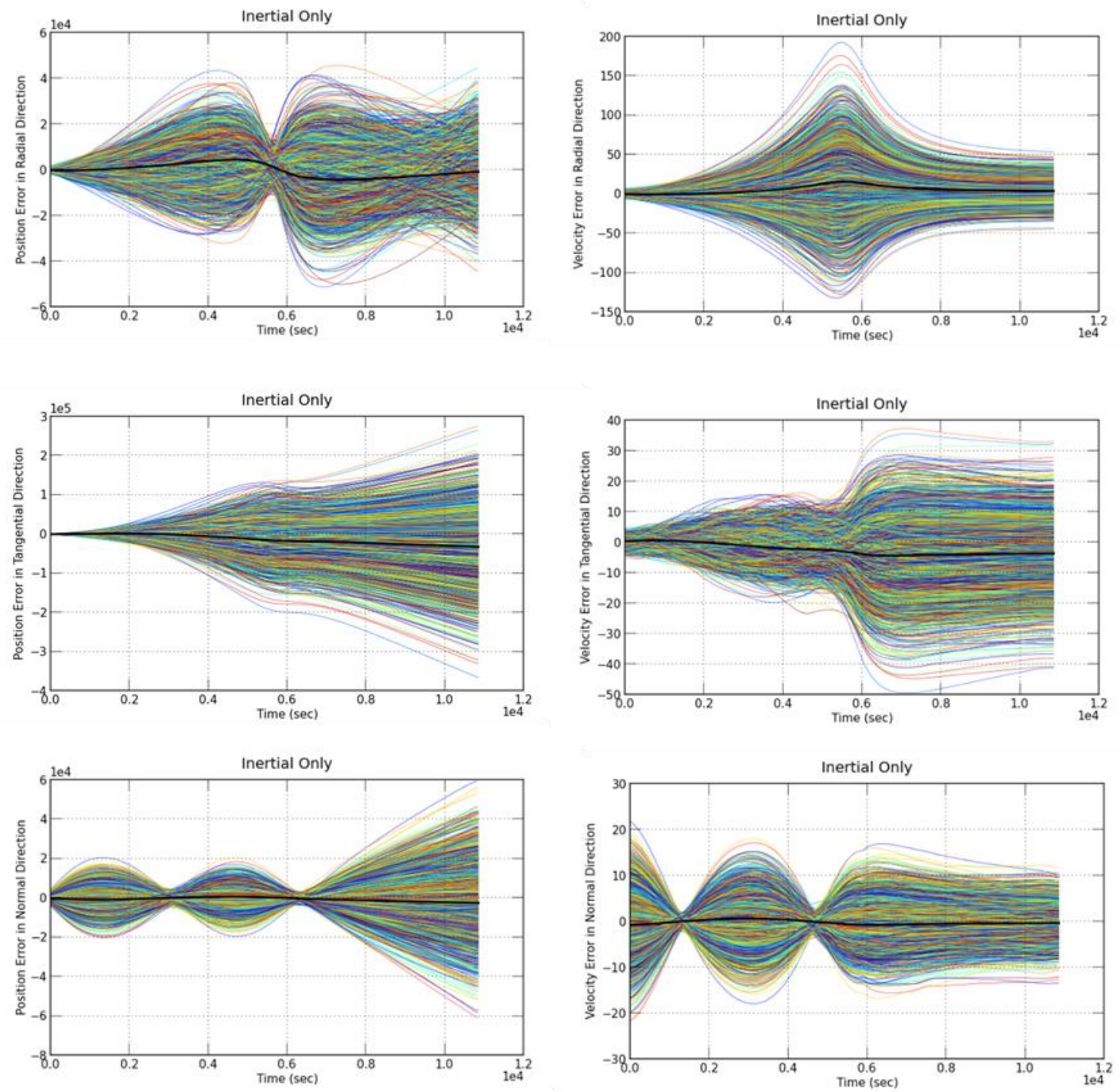

Figure 2: Inertial Error Growth in Space

With these large state dispersions at the time of the burn, a very robust set of orbital targets must be developed to allow for lunar fly-by under a wide variety of scenarios. While the onboard algorithms do support the capability to tune orbital parameters based on time of launch, they do not consider these larger dispersions. To optimize the orbital targets, the nominal state was used in the Copernicus (Williams) optimization tool to minimize delta-velocity of disposal manuever and to achieve enough energy after Lunar fly-by to enter heliocentric space, through the measurement of C3 (a value greater than 0 means that the vehicle has enough energy to leave the Earth-Moon system). This nominal target was then applied to the individual dispersed states (assuming perfect guidance capability) to capture the navigated vehicle capability. Each was then propagated out past Lunar fly-by to assess capability. Figure 3 captures the disposal capability with a notional state-of-the-art inertial navigation architecture. Thus, a positive $\mathrm{C} 3$ is considered a successful disposal. Due to the modeling of the moon as a point mass in this simulation, some of these cases actually impacted the Lunar surface (with minimum distance to the lunar surface less than 0 ). The Lunar footprint of these is shown in Figure 4. It must also be noted that this plot has been cropped to show an area of interest in line with later results. The lines continues up and to the left, with the majority of the Monte Carlo runs. 


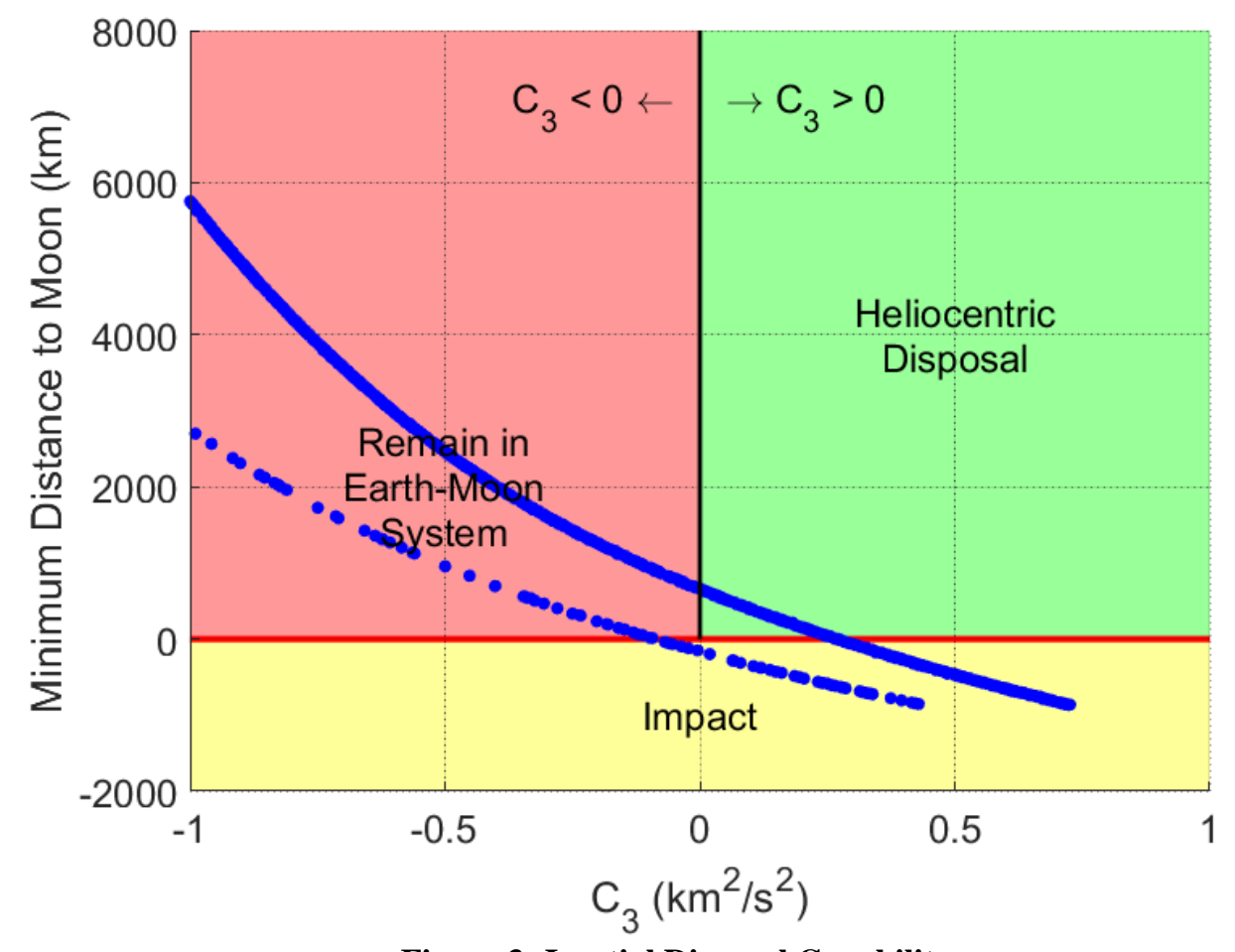

Figure 3: Inertial Disposal Capability

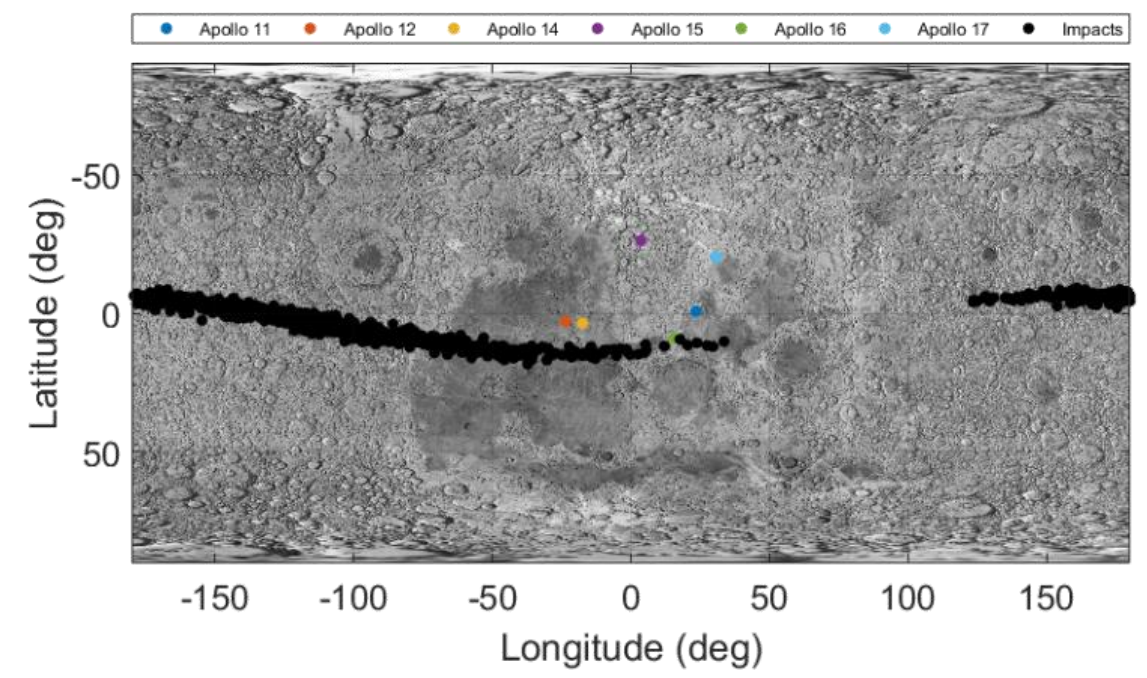

Figure 4: Inertial-only Lunar Footprint

By the time the dispersed navigated states were propagated to the moon, the dispersions had grown to be greater than the diameter of the moon. This is also shown in Figure 3 through the bimodal behavior. The EM-1 mission is a DRO, with the MPCV approaching in front of the moon. As such, the upper bar represents matching this behavior. The bottom line, though, captures cases that flew behind the moon. This behavior is captured well in Figure 1. The integrated results from this analysis shows a probability of $48 \%$ percent chance of successful heliocentric disposal with $42 \%$ chance of lunar impact. Additionally, the figures clearly show the effect of Earth-Moon geometry on the difficulty of this manuever. This is seen in the successful disposals having a very limited window of altitudes between 0 And 500km from the Lunar surface. 


\section{DISPOSAL OPTIONS AND CONSIDERATIONS}

Per NASA-STD-8719-14, a 90\% confidence is required to successfully meet stage disposal requirements. As shown in the above figures, this is very challenging to achieve with the system as defined. As such, the SLS Navigation team performed a design trade to identify potential options for reducing these dispersions through reduction of navigated state errors. Several options for considered to improve the state, with the following primary options: state update from payload, state update from ground, enhanced inertial navigation algorithms and models, GPS-provided state measurements, and alternate mission design. The Orion vehicle has a sophisticated and robust navigation architecture (D'Souza) to enable deep space navigation and could provide a solution. The caveats are that an interface must be established, designed, and implemented between the two elements, requiring a large amount of integration, software and hardware elements. Additionally, providing a state update form the ground was also considered as a potential option, but was not selected due to additional hardware requirements on the upper stage, including a DSN transponder and an expanded uplink capability. Again, this effort was too schedule- and cost-prohibitive to be implemented. Enhanced navigation and gravity models could provide additional capability, but required large software updates and development efforts. In orbit coasts could also be reduced to limit the time for inertial navigation errors to grow. The cost of this is limited checkout and operational time prior to Trans-Lunar Injection and must be carefully traded with operational constraints.

Lastly, the inclusion of GPS was considered. This would allow the stage to have enhanced state knowledge throughout its orbital flight profile, vastly reducing navigated errors and allowing for autonomous operation on orbit. This does presents its own challenges, primarily involving software development, as well as integration and procurement efforts to add a new hardware element to an existing design. Additionally, due to the cost and schedule constraints, the solution space of GPS receivers was limited to those already commercially available. The following section provides more details on how the GPS assessment was performed to understand its capability for this mission scenario.

\section{GPS INTEGRATION APPROACH}

In order to enable proper disposal of the upper stage, the analysis focused on the integration of GPS measurements into the onboard navigation solution. This was chosen to provide a bootstrap capability to the existing navigation system to allow for improvement of navigation capability with minimal changes to existing hardware and systems integration. To enable modeling of this architecture, a generic GPS model was implemented to allow for error assessment and dynamic analysis along the inspace trajectory. Due to the focus of this analysis on requirements definition and systems definition, this model was implemented to allow assessment of the generic capability of a GPS receiver. As opposed to implementation of a highly detailed software model of the receiver and constellation, this sensor model was based on the definition of the GPS service volume and defined performance over orbital bands (LEO, MEO, and HEO) based on representative Errors were then generated using 1sigma dispersions on time, position, and velocity that were in family with the GPS specification and typical performance. A notional latency model was also included to capture the need to integrate with independent inertial observations. The receiver was assumed to output measurements at $10 \mathrm{~Hz}$.

In order to integrate the state observations with the inertially integrated state, a loosely-coupled filter was implemented to allow for generating updates to the estimated state. This filter was implemented as a 15-state Extended Kalman Filter, and included states capturing, position, velocity, attitude, accelerometer bias, and gyroscope error bias terms. The mechanization of the filter is similar to that described in (Groves) and is executed at $1 \mathrm{~Hz}$. The equations of motion were defined in a true of date (TOD) inertial frame, to cocincide with the state integraiton equations. For calculation of gravity, the state was rotatated into an ECEF frame for processing with a model including up to J4 terms. This inertial acceleration was then rotated back into the inertail frame for use in the equations of motion, which include an Euler $2^{\text {nd }}$ order integration method at $100 \mathrm{~Hz}$. The navigation equations and integration technique were chosen to match that typically used in inetial-only systems.

The implementation of accelerometer and gyroscope bias terms is needed due to the long coast periods of the system on-orbit. Any uncorrected sensor errors terms would continue to integrate over hour-long trajectories leading to increased state dispersions. As shown in Figure 2, the integration errors grow to large levels over the in-space trajectory. By using the GPS measurements to support estimation of the errors terms, integration errors due to these sensor uncertainties can be minimized. These inertial error terms were included in the filter dynamics through inclusion of the current rotation between body and inertial frames. In order to reduce uncertainties in the state dynamics models, the covariance was propagated at high rate $(50 \mathrm{~Hz})$ to allow synchronization with the latest attitude solution. This is particularly important at high altitudes as the vehicle navigates outside of the GPS architecture and prior to the final disposal burn. Inclusion of these error correction, allows for reducing 
errors integrated. Additionally, even with limited observability into attitude, using the filter to improve attitude state also helps manage the integrated errors over long flight, helping to improve pointing and targeting capability.

\section{NAVIGATION CAPABILITY WITH GPS AT HIGH ALTITUDE}

In order to assess the navigation capability, a Monte Carlo-based analysis of the in-space trajectory was performed to capture final navigation state dispersions prior to the disposal manuever. The GPS navigation filter was included into the model of the vehicle's onboard GNC algorithms. The initial state dispersions were seeded from results of notional ascent vehicle capability and the upper stage's inertial navigation over ascent. The focus of this analysis was to verify disposal performance with GPS aiding and to determine the lowest altitude needed for coverage. This information helps to define the capability and grade of receiver required to successfully complete the mission. To assess a broad range of GPS outage altitudes, several were modeled: $1000 \mathrm{~km}, 2000 \mathrm{~km}, 4000 \mathrm{~km}, 6000 \mathrm{~km}, 8000 \mathrm{~km}, 10000 \mathrm{~km}, 12000 \mathrm{~km}$, and $14000 \mathrm{~km}$. These were chosen to capture receivers operational at LEO, MEO, and HEO altitudes. These altitudes were modeled as cutoffs in the GPS performance model of last fix. The altitude limit is especially important to the vehicle's large radial velocity coming out of the TLI manuever as shown in Figure 5. Importantly, all of the cases also had pure inertial navigation regions between GPS outage and the disposal maneuver.

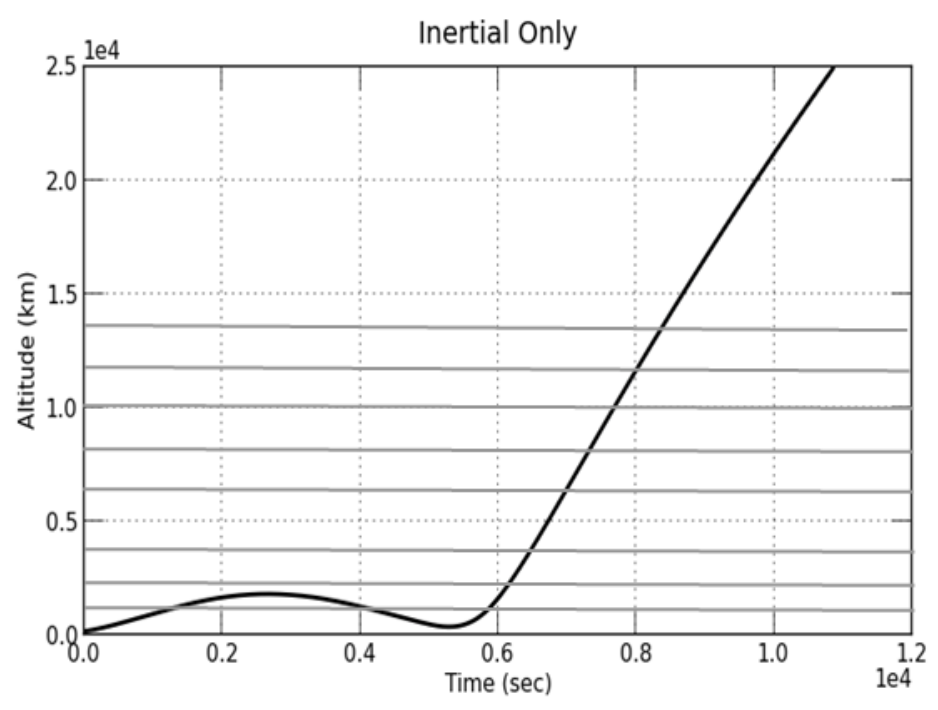

Figure 5: GPS Outage Altitude over Inertial Trajectory

With the addition of GPS aiding, the navigation dispersions were greatly reduced. This allowed for the errors to be maintained at very high accuracy across the entire orbital trajectory, and reduced error growth post GPS outage. Figure 6 provides an overview of how the navigation errors change over the trajectory for a pure inertial flight, $6000 \mathrm{~km}$ outage, and $14000 \mathrm{~km}$. These plots shown position and velocity errors in the Radial direction. First, it's important to note that even use of GPS strictly in LEO greatly reduces the errors at disposal two orders of magnitude. Additionally, the effect of the large altitude rate is also apparent as even though the two bottom cases show very different altitudes, they are reached rather quick comparatively. Important to note is the similar behavior of those two cases in terms of velocity error, and the fact that the increased position uncertainty is a function of simply integrating this error until disposal. This provides insight into the tradeoff between these two variables, and indicates that for the altitudes considered, there is diminishing returns for high altitude cases in terms of improved state knowledge as shown in Figure 7. This figure shows the 1-sigma uncertainty of inertial position and velocity at the disposal manuever in a RTN frame as a function of GPS outage altitude.

In addition to navigation errors, it is also important to assess the performance in terms of integrated guidance and navigation by looking at the total state dispersions (in addition to knowledge uncertainty). Figure 8 displays the mean and 1-sigma state uncertainties in terms of position at the time of disposal maneuver. The figure identifies that the navigated state's primary sensitivity has shifted from knowledge errors to targeting precision. This is an artifact of using targeting metrics tuned to meet insertion errors for the payload as opposed to tuning for the disposal maneuver (which has much stringent requirements as the next section will show). Using GPS greatly improves the mean navigated state closer to the desired target state out of TLI and helps to reduce dispersions. During TLI, the majority of the cases were still in GPS coverage, and as such, the large dispersions 
at disposal indicate further tuning of the guidance can be performed to further reduce the dispersions, For the next section, this research assumes the use of tuned guidance metrics that enable a better match to the navigation knowledge requirements, and allows the research to focus on the effect of state knowledge on navigation uncertainties.
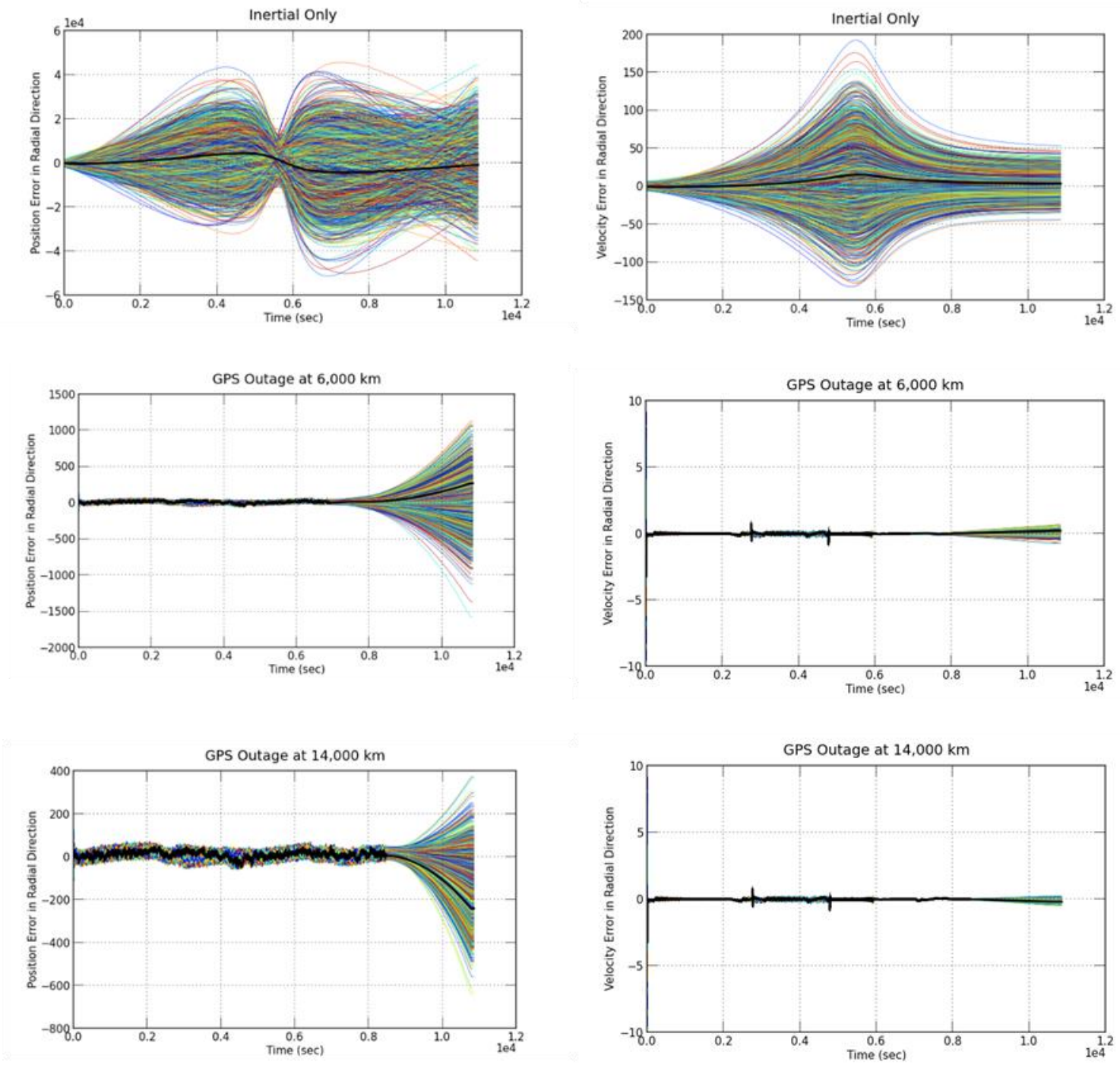

Figure 6: Dynamic Aiding Capability 

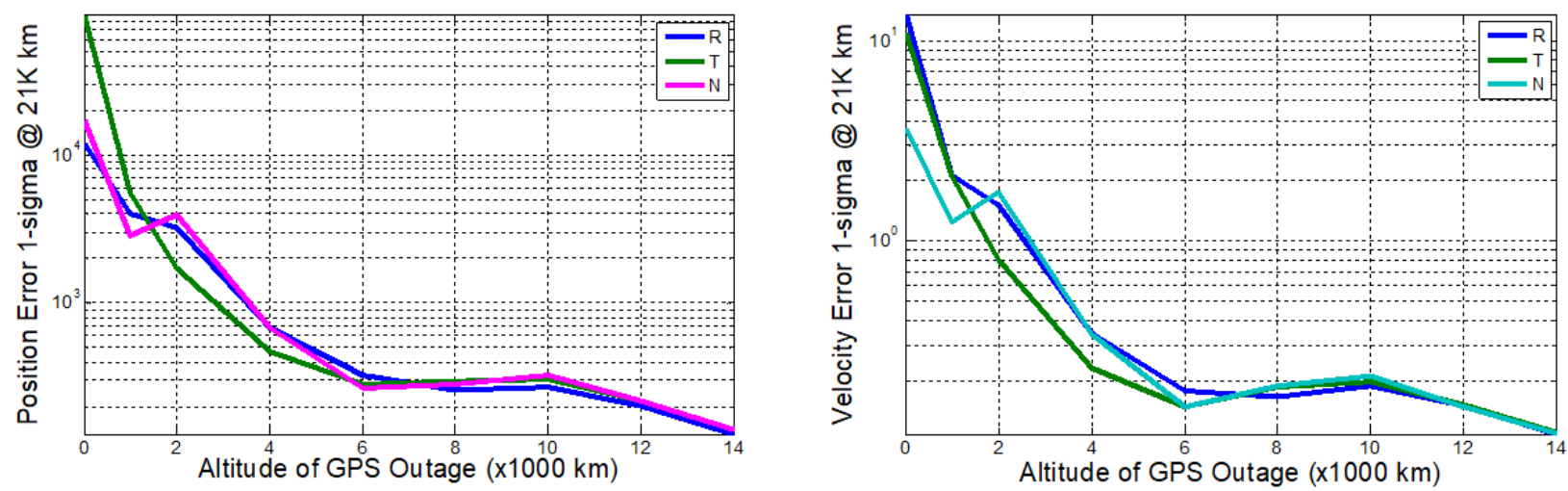

Figure 7: RTN Errors at Disposal
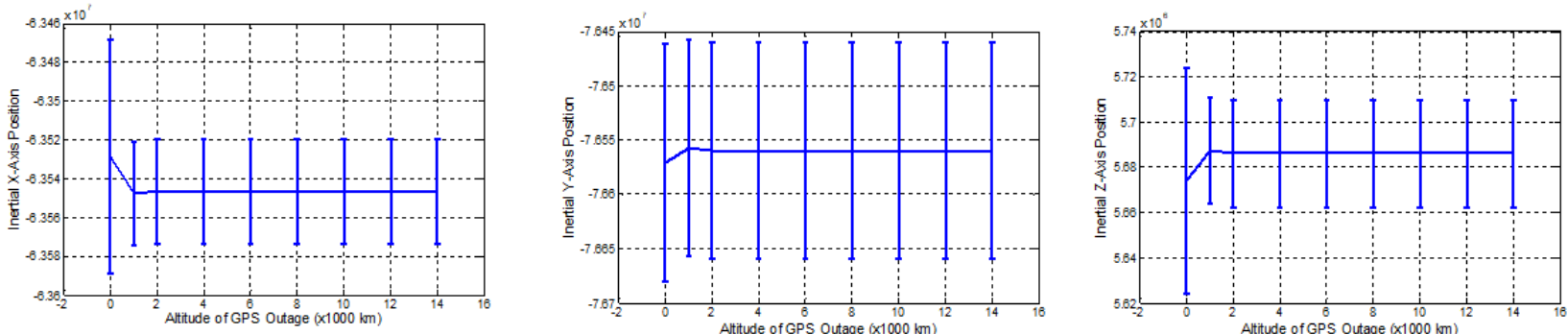

Figure 8: Dispersed Position State at Disposal in an Inertial Frame

\section{GPS-ENABLED DISPOSAL CAPABILITY}

In traditional trajectory and target optimization, errors sources which lead to trajectory dispersions are not taken into account. This leads to targets that were optimized for a minimum delta-velocity and maximum C3 post lunar encounter. Maximizing C3 naturally resulted in implicitly minimizing the perilune altitude of trajectory resulting in a large number of lunar impact cases when trajectory dispersions were considered. It became apparent that trajectory dispersions at the end of disposal maneuver needed to be accounted for to develop a target that considers vehicle uncertainties and real mission design. A robust process for optimizing the disposal trajectory for the vehicle disposal guidance targets was needed to allow for successful disposal.

Initially, an approach of simply adding a constraint to the optimization process for an increased lunar perigee altitude was attempted. This approach was sub-optimal and required iterations between trajectory optimization and computationally expensive 6DOF simulation where the estimated targets were developed and incorporated into the 6DOF simulation as guidance targets. The results of a Monte Carlo analysis were analyzed and the optimization constraints were adjusted. This was time consuming and took many iterations due to non-Gaussian trajectory dispersions near the moon, from the effect of Lunar gravity and the variation in transit time relative to the moon due to the dispersions in the TLI orbit.

A process was needed which optimized the trajectory for the target while considering the trajectory dispersions due to navigation. The approach developed expands the optimization problem to consider the dispersions due to navigation errors at Lunar perigee. The error ellipsoid is known prior to the disposal maneuver but cannot be known precisely after the maneuver due to the fact that the trajectory optimization process affects the disposal maneuver in direction, duration, and magnitude. The constraints on the nominal state plus error ellipsoid are applied to the error ellipsoid that has been propagated to near the lunar surface. The additional constraints are for positive lunar altitude and for positive $\mathrm{C} 3$ beyond the moon.

The following equations describe the propagation of a state and covariance using a state transition matrix, $\phi\left(t_{D S P}, t_{L E}\right)$, to propagate the state from disposal to Lunar encounter.

$$
\begin{gathered}
x_{L E}=\phi\left(t_{D S P}, t_{L E}\right) x_{D S P} \\
P_{L E}=\phi\left(t_{D S P}, t_{L E}\right) P_{D S P} \phi\left(t_{D S P}, t_{L E}\right)^{T}
\end{gathered}
$$


For the optimization problems, the desire is to optimize a disposal maneuver for a nominal target, $x_{D S P}$, while considering the error ellipsoid about the nominal state, $P_{D S P}$, for minimum delta-velocity required subject to constraints placed on the propagated nominal state and dispersed states, represented by $x_{L E}$ and $P_{L E}$. The constraints implemented were for positive C3 10 days post- perigee passage and positive lunar altitude at perilune. For the optimization problem, the process described by the equations above could not be represented. Instead, the mean pre-disposal state was used as a nominal pre-disposal state. A state transition matrix could not be used and linearity could not be assumed so the optimization tool was used to numerically propagate trajectory states. The error covariance could not be numerically optimized. Instead, the error dispersion is represented by the 12 eigenaxis and eigenvalue combinations for the position and velocity error covariance. The reduced trajectory dispersions are applied to the mean state and the optimization problem is expanded to consider 13 trajectories, a single nominal and 12 derived trajectories dispersed from the post-burn nominal trajectory state. The optimization problem was reformulated for a minimum delta-velocity disposal maneuver subject to constraints on post-burn child trajectories for positive lunar altitude and positive $\mathrm{C} 3$.

As seen in previous sections, the error covariance resulting from inertial navigation only is too large to successfully dispose. This results in an infeasible trajectory optimization problem as reformulated. The solution is to add an additional logical loop outside of the optimization problem to scale the eigenvalues used to derive the dispersed child trajectories. Monte Carlo analysis was used to get the initial error covariance. This provides an initial guess of the error and the shape of the error ellipsoid. The eigenvalues were scaled down to develop an initial optimization solution which was feasible and then iteratively scaled up to maximize the size of the error ellipse with respect to feasibility. The resultant nominal post-disposal trajectory state is a robust and approximately optimal disposal target. It approximately represents the optimal disposal target for the maximum error ellipsoid which would result in $100 \%$ heliocentric disposal via Lunar swing-by. To confirm the result, draws are made from the appropriate re-sized error covariance and the errors are applied to the optimal nominal post-disposal state to perform a small Monte Carlo type analysis. The dispersions are propagated in the presence of Earth and Lunar gravity. Other gravitational bodies are considered were determined by previously performed sensitivity analysis. The disposal results are checked for minimum Lunar altitude along the trajectory and C3 post-Lunar encounter.

For the navigation cases described above with variable GPS outage assumed, this process was performed to find the approximate minimum altitude required of GPS measurements to support the heliocentric disposal requirements. As described above, the cases were delineated by the altitude at which GPS measurements were denied to the GPS aided inertial navigation system. The object was to characterize the effect of GPS outage by altitude on disposal probability. For each case below $6000 \mathrm{~km}$, the disposal target was re-optimized to account for the differences in the mean state due to integration error and the state error due to the integration of gravity anomaly. Perturbations in the state post-disposal state affect the nominal disposal orbit which affects the time of arrival at the moon. Time of arrival has a large effect on the dispersion at the lunar perigee due to the lunar dynamics and difference in gravity acting on the spacecraft.

For each of the cases, the dispersed disposal states were taken from 6DOF simulation Monte Carlo analysis. The post-disposal state dispersions were applied to the new optimal target and propagated to the moon to assess minimum lunar altitude and beyond the moon to assess C3 10 days post- Lunar encounter. The results are tabularized in Figure 9. 


\begin{tabular}{|l|c|c|c|}
\hline Monte Carlo Case & \% Lunar Impact & \% Heliocentric & \% Success \\
\hline No GPS & 41.83 & 48.88 & 7.05 \\
\hline GPS Outage @ 1000km & 28.29 & 60.17 & 31.88 \\
\hline GPS Outage @ 2000km & 25.74 & 88.66 & 62.92 \\
\hline GPS Outage @ 4000km & 03.85 & 96.30 & 92.45 \\
\hline GPS Outage @ 6000km & 00.05 & 97.65 & 97.6 \\
\hline GPS Outage @ 8000km & 00.00 & 91.85 & 91.85 \\
\hline GPS Outage @ 10000km & 00.00 & 99.95 & 99.95 \\
\hline GPS Outage @ 12000km & 00.00 & 98.45 & 98.45 \\
\hline GPS Outage @ 14000km & 00.00 & 93.70 & 93.70 \\
\hline
\end{tabular}

Figure 9: Disposal Results for Monte

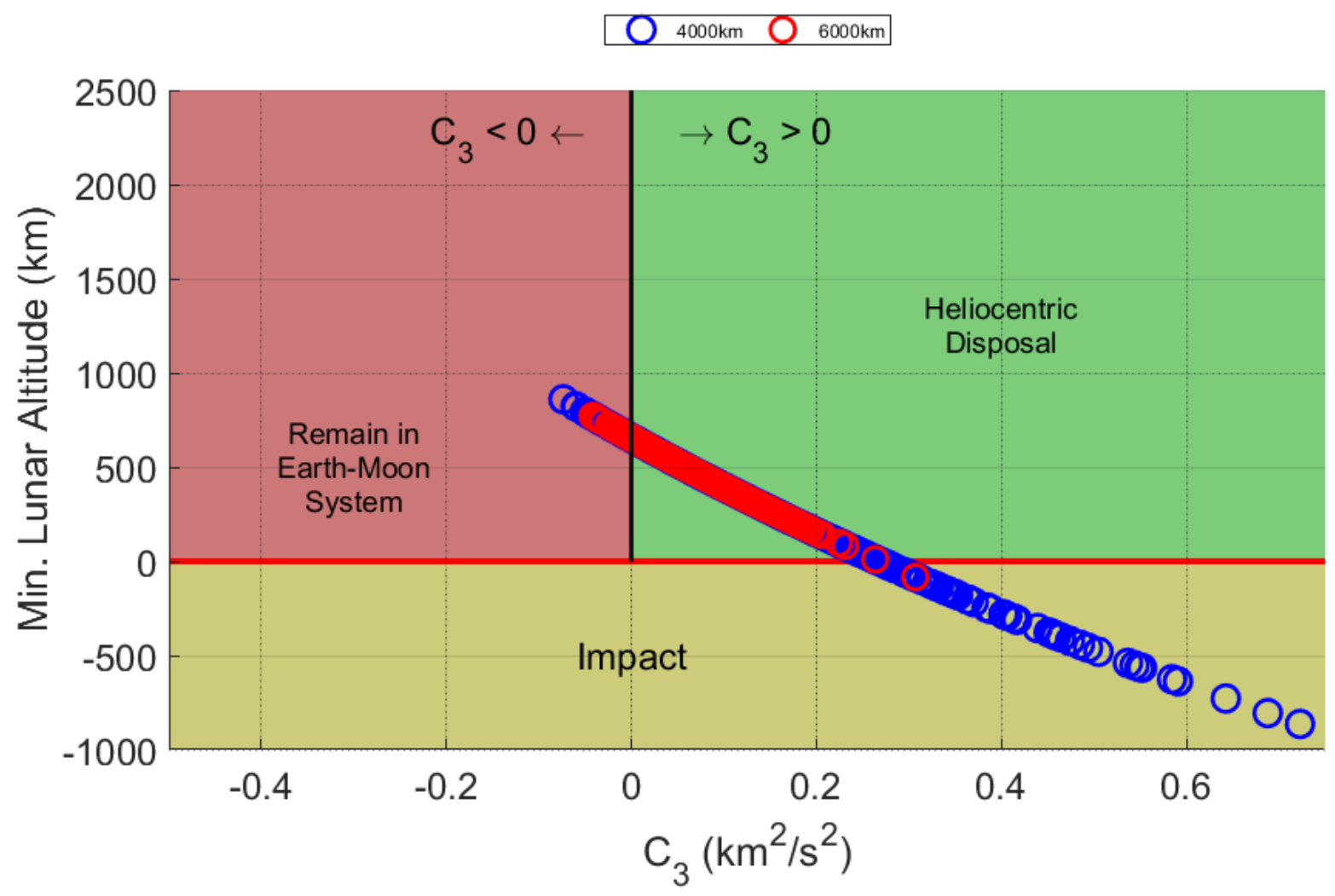

Figure 10: Disposal Result, GPS Altitude at 4000km and 6000km Altitude 


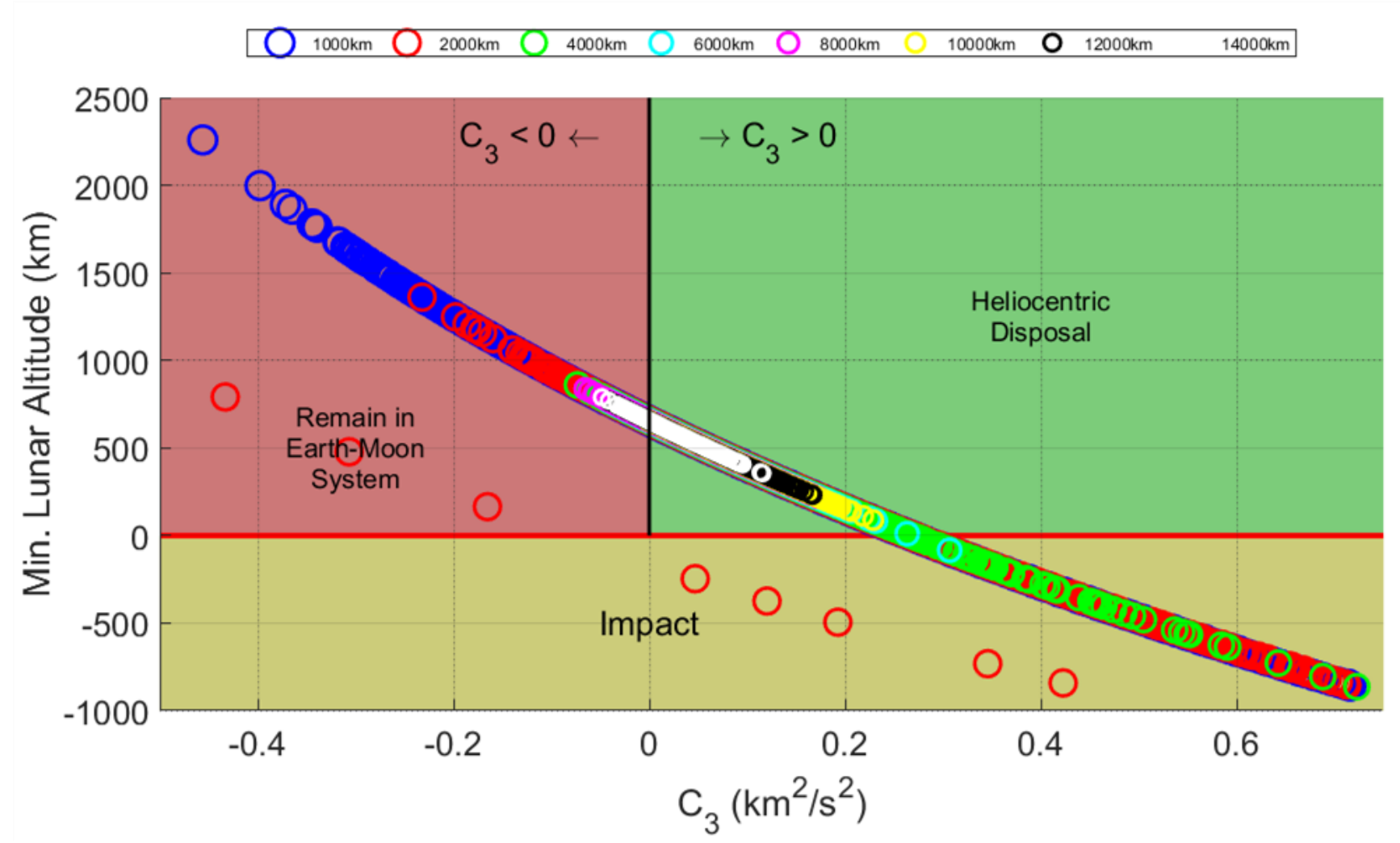

Figure 11: Disposal Result, All Simulated GPS Outage Altitudes

With the optimized targets and considering the trajectory dispersions developed from errors in the navigation states, the table shows that the minimum altitude for GPS availability required to support heliocentric disposal for this specific mission case is approximately $4000 \mathrm{~km}$. Figure 10 graphically depicts the 2000 dispersed cases run for the break point at $4000 \mathrm{~km}$ and $6000 \mathrm{~km}$ altitude GPS outage and where they fall with respect to:

- Lunar impact, negative minimum lunar altitude

- Remaining in Earth-Moon system, negative C3 10 days post- lunar fly-by and positive lunar altitude

- Successful heliocentric disposal, positive C3 at 10 days post- lunar fly-by and positive lunar altitude.

All of the cases are plotted in Figure 11. Note that the higher altitude GPS outage cases were not run with re-optimized targets and that target re-optimization would have served to better center the dispersion within the window for successful heliocentric disposal.

\section{INTEGRATION CHALLENGES AND DISCUSSION}

As shown in this research, the integration of a GPS receiver to allow for state aiding at high altitude provides sufficient performance to meet state disposal requirements. With a potential technical solution in hand, the programmatic caveats must be addressed in how to accomplish this. The use of GPS at these higher altitudes proves difficult simply in terms of availability of receivers. While there is a breadth of receivers that have flight heritage in Low Earth Orbits and several options are available for High Earth Orbit and beyond (W. Banford), the performance capability of these units are not well characterized for flight through these altitudes. These two sets of receivers also have a large price differential as well as altitude performance. While the constellation specification does have expected levels of performance at these altitudes (Global Positioning Systems Directorate), vendors can be hesitant to certify performance due to the limited number of heritage flights and applications in this use case. In addition to in-orbit performance, stringent radiation and environmental survivability requirements must be met for extended operation outside of LEO. This is particularly important for cases where a terrestrial- or LEO-focused receiver is considered. Additionally, as operations for EM-2 (Donahue) and beyond may include additional co-manifested payload missions, the need for high altitude performance is further reinforced as the amount of time in orbit is extended prior to the 
final disposal maneuver, allowing for greater periods of inertial error drift. In addition to specific receiver hardware capabilities, this higher altitude performance is also heavily dependent upon maintaining the existing sidelobes of the GPS (Bauer).

Several other options should be considered and several forward research topics are identified from this work. Designing for the disposal manuever early in mission planning assessments will help to enable a more robust disposal solution. Similarly, continued tuning of the guidance algorithms is needed to match the enhanced navigation capability due to GPS aiding. Additionally, this research did not fully address timing uncertainties. This approach assumed development of an externally provided disposal state for the guidance algorithms based on the time of maneuver and state dispersions. Further enhanced capability is enabled by moving the target generation process onboard the spacecraft, allowing for real-time targeting based on the planned timing of the maneuver and the vehicle's current state. This will allow for further improvement in probability of success of the disposal maneuver and provide enhanced capabilities necessary for future human spaceflight missions, allowing for increased autonomy from Earth-based systems, allowing for more responsive and robust mission architectures. The lessons from this research are being applied and algorithms matured for inclusion into the design and analysis of the Exploration Upper Stage for EM-2.

\section{ACKNOWLEDGMENTS}

The authors would like to acknowledge the support of Robert Stough (NASA/MSFC) for his contributions to the initial development of the disposal assessment, as well as the support of the SLS VM EDLE, Joey Broome (NASA/MSFC) for his funding of this research and presentation. Thanks also go to the developers of Copernicus, Jacob Williams and Juan Senent, for their instruction and for continuing to provide the capability to interface with Copernicus through an API. Lastly, credit is due to our branch and division management, Heather Koehler and Don Krupp, for their continued support of our design, analysis, and publication activities. Furthermore, Emerson Oliver would like to acknowledge the support of his skill lead Joe Groszkiewicz and manager Jimmy Compton for their continued support.

\section{REFERENCES}

Bauer, F.H and Moreau, M.C. "The GPS Space Service Volume." ION GNSS 2006. Fort Worth, TX, 2006.

Donahue, B. and Sigmon, S. "Space Launch System: Block 1B Configuration: Development and Mission Opportunities." 53rd AIAA/SAE/ASEE Joint Propulsion Conference. Atlanta, GA, 2017.

D'Souza, G. N. Holt and C. "Orion Absolute Navigation System Progress and Challenges." AIAA Guidance, Navigation, and Control Conference. Minneapolis, MN, 2012. AIAA-2012-4995.

Gerstenmaier, W.H. Human Exploration \& Operations Update on Mission Planning for NAC. 30 November 2016.

Global Positioning Systems Directorate. "Navstar GPS Space Segment/Navigation User Interfaces." 24 September 2013. www.gps.gov/technical/icwg/IS-GPS-200H.pdf.

Groves, P.D. Principles of GNSS, Inertial, and Multisensor Integrated Navigation Systems. Artech House, 2013.

Hanson, J.M and Hall, C.E. "Learning about Ares I from Monte Carlo Simulation." AIAA Guidance, Navigation, and Control Conference and Exhibit. Honolulu, HI: AIAA, 2008. AIAA-2008-6622.

Honeywell Aerospace Electronic Systems. "Redundant Launch Vehicle Guidance." September 2003. https://aerocontent.honeywell.com/aero/common/documents/RLVG.pdf. September 2017.

NASA. "Process for Limiting Orbital Debris." NASA-STD-8719.14A. 2012.

W. Banford, B. Naasz, and M.C. Moreau. "Navigation Performance in High Earth Orbits using Navigator GPS Receiver." 29th Annual AAS Guidance and Control Conference. Breckenridge, CO, 2006.

Williams, Jacob. "A New Architecture for Extending the Capabilities of the Copernicus Trajectory Optimization Program." AIAA/AAS Astrodynamics Specialist Conference. Vail, CO, 2015. 